\title{
SAND- $-90-2558 \mathrm{C}$ \\ DE9 1004897
}

INTERFACIAL FORCE SENSOR WITH FORCE-FEEDBACK CONTROL

Stephen A. Joyce, J. E. Houston, and Bradley K. \$Smith

Sandia National Laboratories

Albuquerque, NM 87185 USA

ABSTRACT

A new interfacial. force microscope capable of measuring the forces between two surfaces over the entire range of surface separations, up to contact, has been developed. The design is centered around a differential capacitance displacement sensor where the common capacitor plate is supported by torsion bars. A force-feedback control system balances the interfacial forces at the sensor, maintaining the common capacitor plate at its rest position. This control eliminates the instability which occurs with the conventional cantilever-based force sensors when the attractive force gradient exceeds the mechanical stiffness of the cantilever. The ability to measure interfacial forces at surface separations smaller than this instability point using the feedback control is demonstrated.

INTRODUCTION

The atomic force microscope (AFM) has become a popular technique for the imaging of both conducting and insulating surfaces on a microscopic scale (1). In addition to its imaging capability, the AFM also can measure the interfacial forces as two surfaces arf brought together. A knowledge of the forces present between two surfaces is of fundamental importance to any detailed understanding of many phenomena such as adhesion, fracture, and tribology. In this light, it is somewhat surprising that there have been only a few experimental studies wich specifically address the nature of 
these interfacial forces with an AFM (2-7).

As normally configured, the AFM consists of a fixed sample and a tip mounted on a cantilever beam of known stiffness. Interfacial forces are determined by measuring the

deflection of the cantilever while in the proximity of the fixed sample. An instability in the cantilever-tip-sample potential occurs when the tip-sample force gradient, $\partial F / \partial z$, where $z$ is the surface separation, exceeds the force constant of the cantilever. This instability results in the discontinuous movement of the probe into virtual contact with the sample. This behavior, commoniy referred to as "jumping", has been described in detail by others (8). For many systems of interest, jumping may occur at surface separations of from a few $\AA$ up to hundreds of $\AA$ depending on the stiffness of the spring and the nature of the interfacial forces. The instability therefore precludes measurement of the interfacial forces over a broad range of separations, the region of greatest significance to a detailed characterization of surface-surface interactions.

In tilis paper, we describe a new sensor for measuring interfacial forces which incorporates force-feedback control and avoids the instability associated with conventional techniques. This scheme permits the determination of interfacial forces over the entire range of surface separations, from very large up to actual repulsive contact.

\section{CONCEPT}

The instability encountered in deflection force sensors occurs when $\partial F / \partial z$, the force gradient between probe and sample, exceeds $k$, the sensor force constant. The instability can be avoided, however, by balancing the interfacial force on the probe by feeding back an equal and opposite force. The net result is to keep the probe's position rigidly fixed in space as the sample is moved into proximity. 'There have been two previously published reports of force-balance schemes employed to measure interfacial forces $(3,9)$. 
In the first method, the initial displacement of the probe is monitored using optical interference techniques and then counterbalanced by inductive forces (9). The second method employs the tunneling current from a second tip located behind the cantilever to measure displacements and moves the cantilever support to keep the probe fixed at the same position in space, effectively varying the spring constant (3). We have developed a new force balance based on a differential capacitance sensor which has the unique feature that the capacitor acts both as the displacement detector, through changes in capacitance, and as the counterbalance by the application of a restoring electrostatic force.

In a typical capacitance transducer, one plate is fixed while the other is free to move in response to an external force. The movement of the free plate changes the capacitor spacing or gap and therefore results in a change in the capacitance. A feature which, to our knowledge, has not been previously exploited is the ability to balance the forces on the free plate by applying a voltage, $V$, to the fixed plate. The voltage on the fixed plate results in an electrostatic force, $F_{C}$, on the free plate, where $F_{C}=C V^{2} / 2 d$ for a parallel plate capacitor.

Note that the electrostaicic force is always attractive. Thus only external forces, which are in opposition to the capacitance force, can be counterbalanced. We use a differential capacitor to avoid this limitation. The electrostatic force can on $1 y$ act as a restoring force when the capacitor gap increases, as will be the case when the interfacial force is attractive. With a differential capacitor, net repulsive forces on one capacitor, which cause the gap to decrease, can be counterbalanced by applying the restoring force to the other capacitor where the gap has increased. The differential capacitance sensor in our design is comprised of two fixed plates above which is suspended a cormon plate, (a "teeter-totter"), free to rotate about supporting torsion bars. A schematic is shown in Fig. 1. 
A differential capacitance scheme lends itself naturally to use in a balanced AC bridge. As a sample is brought into proximity of a tip mounted on one half of the teeter-totter, the interfacial forces between tip and sample cause a deflection, $\Delta d$, at that end of the common plate. $\Delta d=F_{i} / k$, where $F_{i}$ is the interfacial force and $k$ is torsional force constant of the teeter-totter. The change in the gap, $\Delta a$, will unbalance the bridge. The bridge output is proportional to $\Delta \mathrm{c} / \mathrm{c}=\Delta \mathrm{d} / 2 \mathrm{~d}$ so long as $\Delta \mathrm{d} \ll \mathrm{d}$. The bridge imbalance can be monitored and an electronic feedback controller can rebalance the bridge by applying a DC voltage to one of the fixed plates. The feedback prevents the jumping by maintaining the common plate at its rest position and electronically determines the interfacial force through the magnitude of the applied DC voltage.

\section{DESIGN}

Two designs for the sensor, which differ primarly in the degree of sophistication, are currently under development. The first, presesntly in use, is fabricated using standard thin film techniques. The base, consisting of the two fixed plates and a ground plane, is made by 1) depositing $200 \AA$ $\mathrm{Cr} / 1000 \AA \mathrm{Au}$ on a glass plate, 2) photomasking, and 3 ) wet etching. The common capacitor plate is etched from $50 \mu$ thick Be-cu sheet. The gap is determined by $50 \mu$ mica shims glued between the base and upper plate. The individual capacitor plate areas are $10 \mathrm{~mm}^{2}$, yielding capacitances of $1.5 \mathrm{pF}$. The torsion bar are $6.3 \mathrm{~mm} \times 150 \mu \times 50 \mu$, giving a calculated force constant, $k, 13 \mathrm{~N} / \mathrm{m}$ and a resonant frequency of $175 \mathrm{~Hz}$.

The second sensor utilizes silicon micromachining techniques. Fabricated from chemical vapor deposited (CVD) polycrystalline silicon, the sensor measures $100 \times 200 \mu$ with torsion arms that are $4 \times 40 \times 2 \mu$. Fabrication requires 5 separate masking levels. The base is formed from $2000 \AA$ of phosphorous-doped polysilicon over an insulating silicon njtride layer. The movable capacitor plate is formed from $2 \mu$ thick polysilicon for stiffness and low residual stress. The capacitor spacing 
is defined by a $0.5 \mu$ sacrificial layer of phosphorous-doped glass, which is later removed with HF solutions. A micromachined sensor bonded to a TO-5 header is shown in figure 2. The individual capacitance is $\sim 0.4 \mathrm{pF}$. The calculated torsional force constant and the resonant frequency are $1 \mathrm{~N} / \mathrm{m}$ and $30 \mathrm{kHz}$, respectively.

In operation, the sensor is configured as a high frequency (typically $2.5 \mathrm{MHz}$ ) capacitor bridge. The bridge output, conditioned by a preamplifier (10), is detected by a PAR 5202 lock-in amplifier, operating at a bandwidth of $1 \mathrm{KHz}$. The feedback circuit is a simple proportional integral derivative controller, which is unique only in its output circuitry. As mentioned above, the magnitude of the electrostatic restoring force varies as $\mathrm{V}^{2}$ and therefore does not depend on the sign of the voltage. The sign of the imbalance voltage is important, however, in determining to which fixed plate the DC voltage is to be applied. This is accomplished through the use of two active diode clamps on the output stage of the feedback controller.

Signal-to-noise considerations indicate that the electronic noise should be dominated by the first transistor stage of the preamplifier. This electronic noise results in force and displacement sensitivities of $0.04 \AA$ and $5 \times 10^{-1} 1_{\mathrm{N}}$, respectively for the thin film fabricated sensor. These numbers should be considered lower limits, as other intrinsic, nonelectronic noise sources, such as the thermal motion of the teeter-totter, will increase these values.

\section{PERFORMANCE}

Several tests were performed to determine the feasibility and capabilities of the differential capacitance sensor and the force-feedback control. The first studies, mechanical and electrostatic force displacement tests, were performed to determine the linearity and sensitivity of the thin film fabricated sensor. The bridge responses to the deflections are, as expected, linear. The minimum detectable deflection 
and force for a $2 \mathrm{KHz}$ bandwidth are on the order of $5 \AA$ and $10^{-8} \mathrm{~N}$, respectively. The deflection and force sensitivities are correlated and are both presently limited by electrical interference. With current noise levels, the micromachined sensor, due to its smaller gap and force constant, would have sensitivities of $0.05 \AA$ and $10^{-12} \mathrm{~N}$. Presumably, better performance can be attained by careful attention to shielding against electrical noise.

The ultimate test of the sensor is, of course, its ability to measure interfacial force profiles and in particular to eliminate the mechanical instability discussed earlier. The interfacial forces were measured with a polymethyl methacrylate (PMMA) sample mounted on a piezo tube scanned in proximity to a platinum tip attached to one end of the $\mathrm{Be}-\mathrm{Cu}$ sensor. These tests were done in air so that both the tip and sensor are undoubtedly covered with oxide and water films. Scans were performed with and without feedback control. Shown in Fig. 3a. are the force profiles as measured by the feedback output as the tip approaches and withdraws from the surface. The long range nature of the forces and the appreciable hysteresis between approach and withdrawal suggest a water capillary interaction (11). The bridge imbalance/sensor displacement, not shown, indicates that the feedback maintains the sensor at its equilibrium position within the noise level throughout the entire scan.

The sensor displacement for the same system measured without feedback is also shown in Fig. 3b. Upon approach, the teeter-totter deflects in response to the interfacial force. At a separation of $\sim 15 \mathrm{~nm}$, the force gradient exceeds the force constant and the teeter-totter jumps into virtual contact with the tip. Further scanning simply results in a mechanical deflection of the sensor and a one-to-one relationship between piezo displacement and bridge imbalance. There is significant hysteresis and a second jumping event upon withdrawal. This type of hysteresis has been observed and detailed by others $(5,6)$. It simply reflects that upon withdrawal the jumping condition does not occur until $\mathrm{k \Delta d}$ is nearly equal to the 
peak attractive force. The above results clearly demonstrate that without feedback control, measurements of the interfacial forces over a $-15 \mathrm{~nm}$ range of surface separations are not be possible.

CONCLUSIONS

We have described the concept of a new interfacial force sensor which does not suffer from the mechanictl instabilities common to deflection-based force sensors. The design and performance of the sensor are presented. The thin film prototype has force and displacement sensitivities of $\sim 10^{-8} \mathrm{~N}$ and $\sim 5 \AA$, currently limited by electrical noise. These numbers are adequate for many interesting systems, although they can be readily improved by careful attention to minimizing circuit noise. Even with current noise levels, the performance of the micromachined sensor should be several orders of magnitude better. Preliminary results demonstrate the sensor's ability to measure interfacial forces at surface separations smaller than the instability point.

\section{ACKNOWLEDGEMENT'S}

The authors wish to thank T. Michalske, T. Klitsner, and G. McClelland (IBM-Almaden) for many helpful discussions and $B$. Lammie and A. Ricco for the making the thin film depositions.

\section{REFERENCES}

(1) G. Binnig, C.F. Quate, C. Gerber, Phys. Rev. Lett. 12, $930(1986)$.

(2) N.A. Burnham, D.D. Domjnguez, R.I. Mowery, R.J. Colton, Phys. Rev. Lett. 64, 1931 (1990).

(3) P.J. Bryant, H.S. Kim, R.H. Deeken, Y.C. Cheng, J. Vac. SCi. Technol. A8, 3502 (1990).

(4) R. Erlandsson, G. Hadziloannou, C.M. Mate, G.M. 
McClelland, S. CHiang, J. Chem. Phys. 89, 5190 (1988)

(5) Y. Martin, C.C. Williams, H. K. Wickramasinghe, J. Appl. Phys. 61, 4723 (1987).

(6) E. Meyer, H. Heinzelmann, P. Grutter, T. Jung, H.R. Hibder, H. Rudin, and H.J. Guntherodt, Thin Solid Films 181, 527 (1989).

(7) A.I. Weisenhorn, P.K. Hansma, T.R. Albrecht, C. F. Quate, Appl. Phys. Lett. 54, 2651 (1989).

(8) N.A. Burnham, R.J. Colton, J. Vac. Sci. Technol. A7, 2906 (1989).

(9) B.V. Derjaguin, V.I. Rabinovich, N.V. Churaev, Nature $272,313(1978)$.

(10)G. Neubauer, S.R. Cohen, G.M. Mcclelland, D. Horne, and C.M. Mate, Rev. Sci. Instrum. 61, 2296 (1990).

(11) J.N.Israelachvili, Intermolecular and surface Forces (Academic Press, London, 1985).

\section{Figure Captions}

Figure 1. Schemaic of the differential capacitance force sensor.

Figure 2. The silicon micromachined sensor mounted on a TO-5 header. The differentially capacitor is the small dark rectangle in the center of the photogragh.

Figure 3. Sensor response with and without feedback for the Pt/PMMA system. (a) The actual force profiles measured with the feedback on. (b) The bridge imbalance in the absence of feedback. 


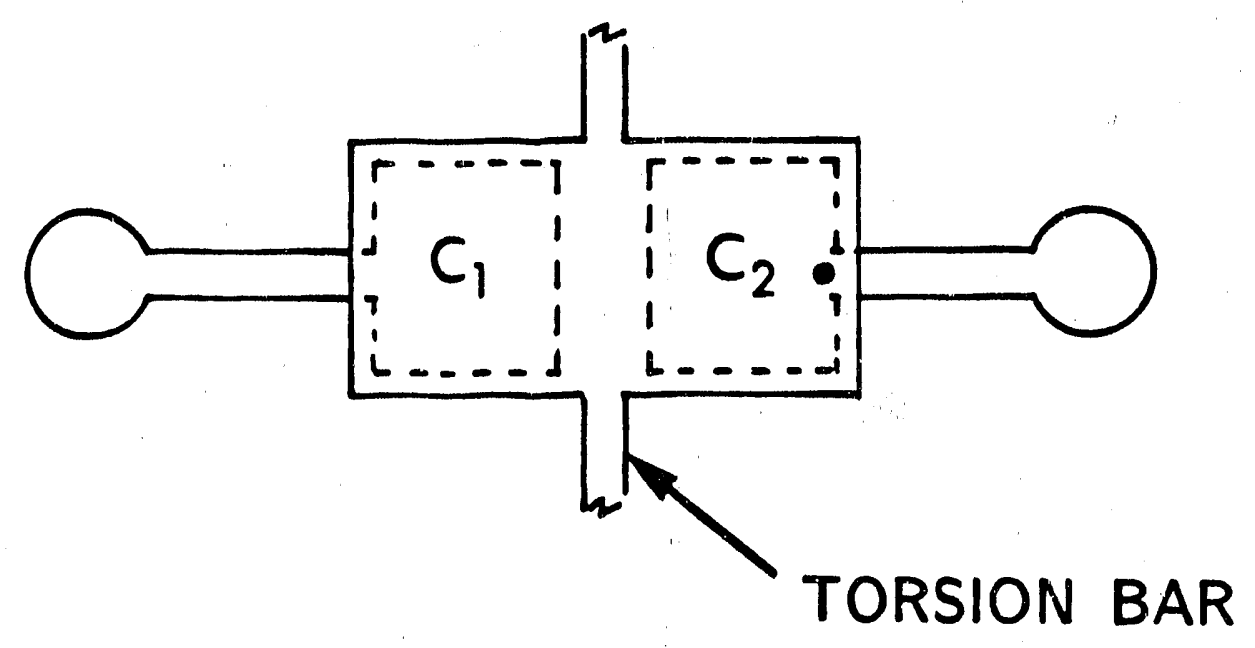

SAMPLE

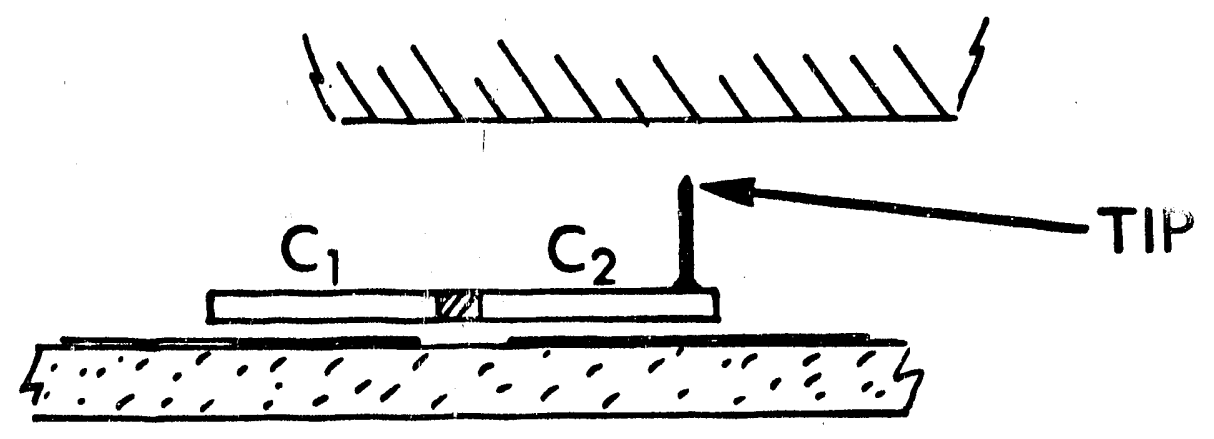




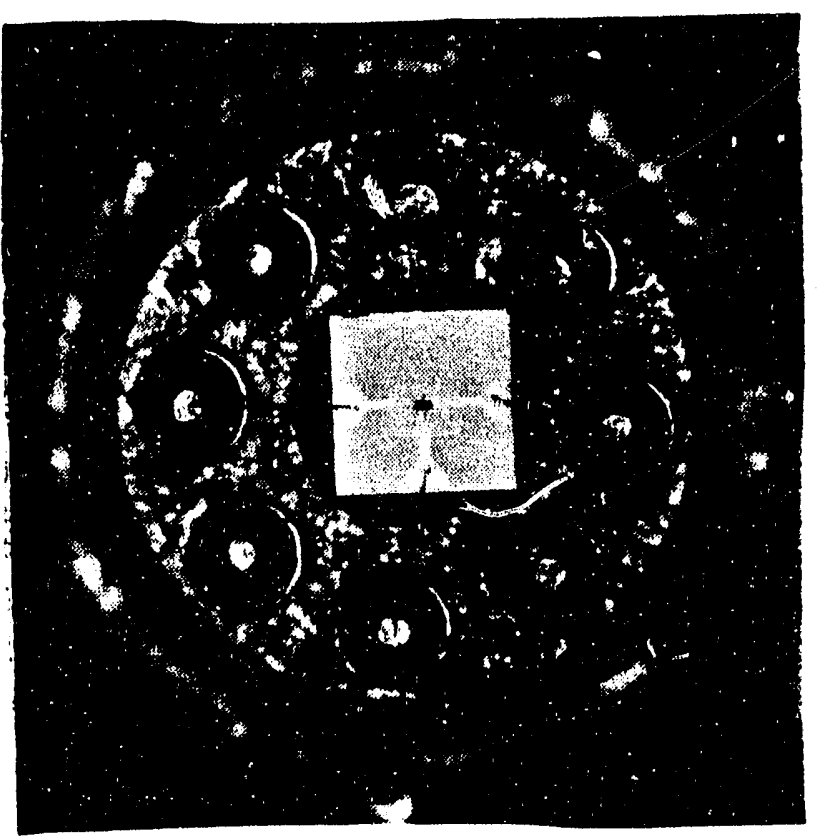

Figz 


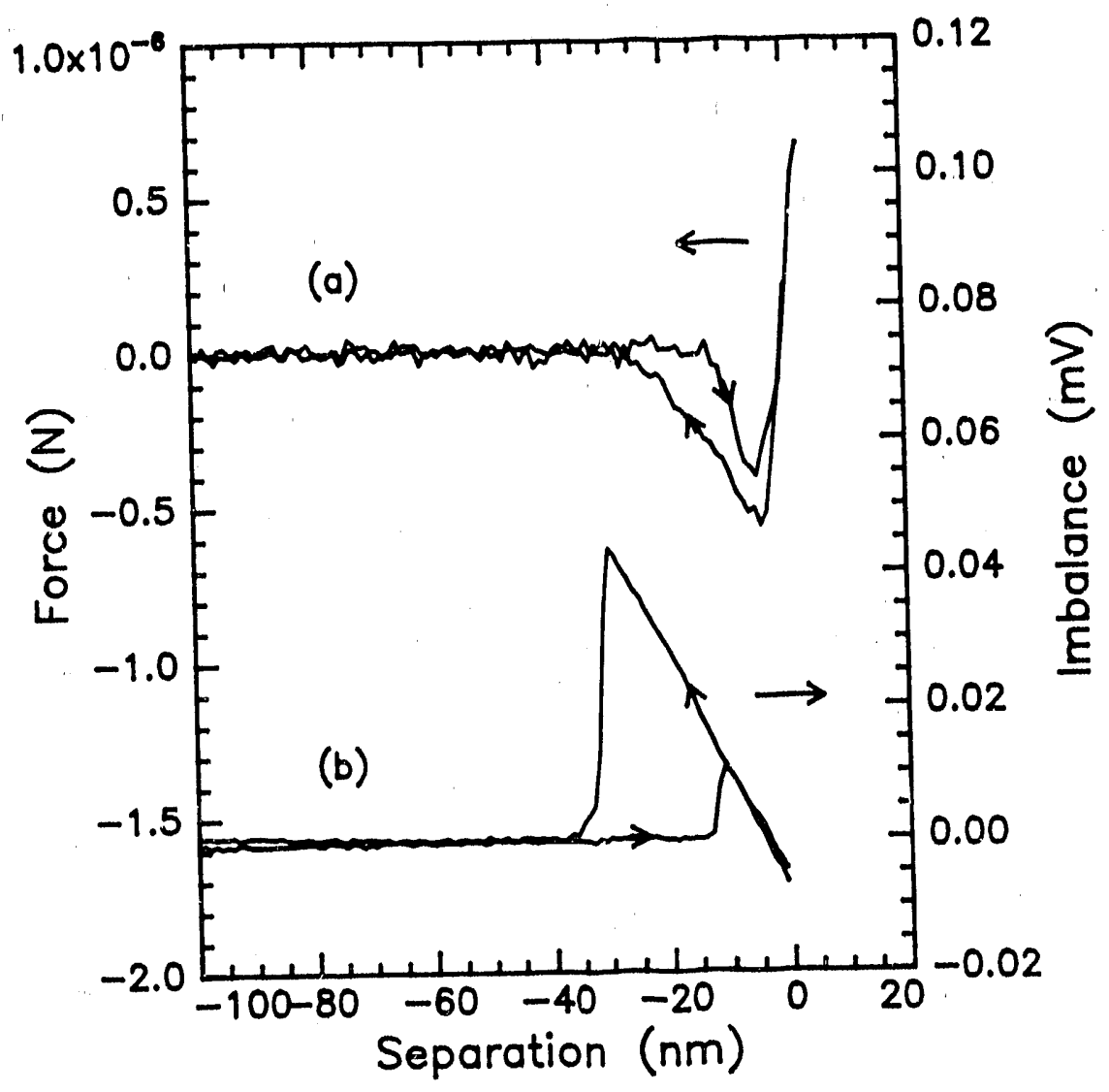

DISCLAIMER

This report was prepared as an account of work sponsored by an agency of the United States Government. Neither the United States Government nor any agency thereof, nor any of their employees, makes any warranty, express or implied, or assumes any legal liability or responsibility for the accuracy, completeness, or usefulness of any information, apparatus, product, or process disclosed, or represents that its use would not infringe privately owned rights. Reference herein to any specific commercial product, process, or service by trade name, trademark, manufacturer, or otherwise does not necessarily constitute or imply its endorsement, recommendation, or favoring by the United States Government or any agency thereof. The views and opinions of authors expressed herein do not necessarily state or reflect those of the United States Government or any agency thereof. 

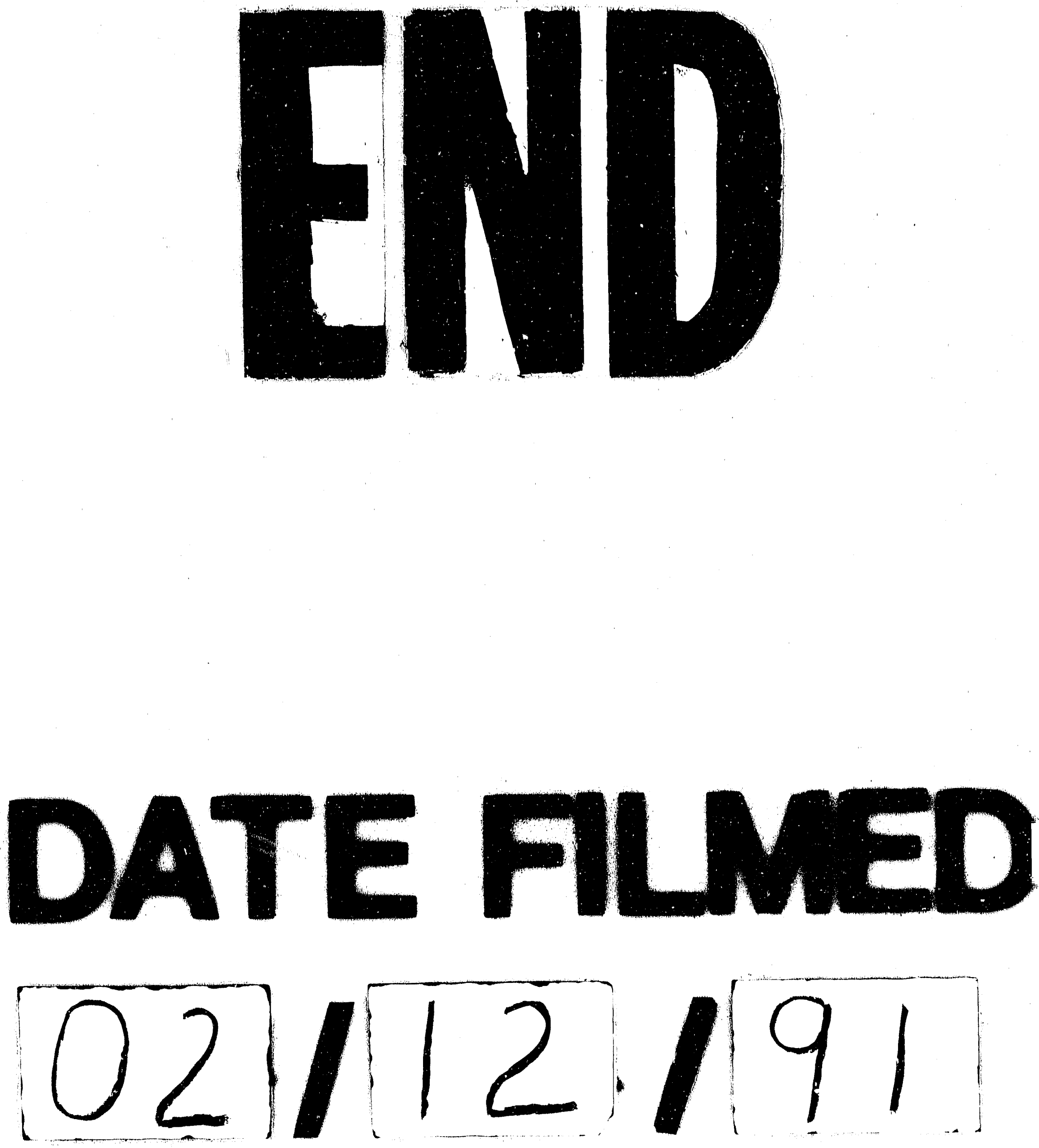